\title{
Expression of MMP-2 and MMP-9 in the rat trigeminal ganglion during the development of temporomandibular joint inflammation
}

\author{
G.C. Nascimento ${ }^{1}$, E. Rizzi ${ }^{2}$, R.F. Gerlach ${ }^{3}$ and C.R.A. Leite-Panissi ${ }^{1,3}$ \\ ${ }^{1}$ Programa de Pós-Graduação em Psicobiologia, Faculdade de Filosofia, Ciências e Letras de Ribeirão Preto, \\ Universidade de São Paulo, Ribeirão Preto, SP, Brasil \\ ${ }^{2}$ Departamento de Farmacologia, Faculdade de Medicina de Ribeirão Preto, Universidade de São Paulo, \\ Ribeirão Preto, SP, Brasil \\ ${ }^{3}$ Departamento de Morfologia, Fisiologia e Patologia Básica, Faculdade de Odontologia de Ribeirão Preto, \\ Universidade de São Paulo, Ribeirão Preto, SP, Brasil
}

\begin{abstract}
Orofacial pain is a prevalent symptom in modern society. Some musculoskeletal orofacial pain is caused by temporomandibular disorders (TMDs). This condition has a multi-factorial etiology, including emotional factors and alteration of the masticator muscle and temporomandibular joints (TMJs). TMJ inflammation is considered to be a cause of pain in patients with TMD. Extracellular proteolytic enzymes, specifically the matrix metalloproteinases (MMPs), have been shown to modulate inflammation and pain. The purpose of this investigation was to determine whether the expression and level of gelatinolytic activity of MMP-2 and MMP-9 in the trigeminal ganglion are altered during different stages of temporomandibular inflammation, as determined by gelatin zymography. This study also evaluated whether mechanical allodynia and orofacial hyperalgesia, induced by the injection of complete Freund's adjuvant into the TMJ capsule, were altered by an MMP inhibitor (doxycycline, DOX). TMJ inflammation was measured by plasma extravasation in the periarticular tissue (Evans blue test) and infiltration of polymorphonuclear neutrophils into the synovial fluid (myeloperoxidase enzyme quantification). MMP expression in the trigeminal ganglion was shown to vary during the phases of the inflammatory process. MMP-9 regulated the early phase and MMP-2 participated in the late phase of this process. Furthermore, increases in plasma extravasation in periarticular tissue and myeloperoxidase activity in the joint tissue, which occurred throughout the inflammation process, were diminished by treatment with DOX, a nonspecific MMP inhibitor. Additionally, the increases of mechanical allodynia and orofacial hyperalgesia were attenuated by the same treatment.
\end{abstract}

Key words: Persistent temporomandibular inflammation; Matrix metalloproteinases; Trigeminal ganglion

\section{Introduction}

Orofacial pain is a very common problem in modern society and is one of the primary reasons for seeking dental treatment in the general population. Orofacial pain originates from conditions associated with hard and soft tissues of the head, face, neck, and all intraoral structures (1).

Musculoskeletal orofacial pain is sometimes caused by temporomandibular disorders (TMDs). This condition has a multi-factorial etiology, including alterations of the masticator muscle, temporomandibular joints (TMJs), and emotional factors (1). In addition, TMJ-associated inflammation is considered to be one of the reasons for the pain reported by TMD patients $(1,2)$. Inflammation can occur in the synovial membrane (i.e., synovitis) or the capsule (i.e., capsulitis) and may result from local trauma, infection or degeneration, changes in collagen formation, or systemic polyarthritis, such as rheumatoid arthritis (3).

Intraarticular administration of complete Freund's adjuvant (CFA), mustard oil, formalin, or adenosine triphosphate (ATP) is used to create animal models of TMJ inflammation (4,5). Among these agents, CFA is 
considered to be a reliable irritant, capable of producing long-term inflammation at the injection site and generating acute and chronic inflammation (2). In addition, TMJ inflammation is associated with deep tissue pain that is often referred to the cutaneous site (6). Furthermore, hyperalgesia and mechanical allodynia induced by the injection of CFA into the TMJ can be evaluated through the facial skin site without invasive manipulations (7).

Studies have shown that macromolecules in the extracellular matrix (ECM) can participate in the inflammatory process. Specifically, the involvement of extracellular proteolytic enzymes locally secreted by cells, such as metalloproteinases and serine proteases, has been shown (8). In fact, there is evidence that matrix metalloproteinases (MMPs) play a major role in the physiological resorption of collagen and other macromolecules during development, postnatal tissue remodeling, and pathological resorption associated with malignant tumors, periodontal disease, and joint destruction in rheumatoid arthritis (9).

MMPs are a family of more than 20 metalloenzymes that cleave several ECM components, including interstitial collagen, basement membranes, fibronectin, laminin, and proteoglycans, thereby releasing fragments with specific biological activities. MMP-9 and MMP-2 are the two most studied MMPs, and the two most frequently expressed MMPs in the nervous system (10). Under basal conditions, MMPs are expressed at very low levels in most tissues, and only a few are expressed in the unstimulated brain (10).

There is strong evidence that MMPs play a critical role in inflammation through the cleavage of ECM proteins, cytokines and chemokines (11), clearly showing that MMP-9 is involved early in the recruitment cascades of neutrophils and that MMP-9 blockade is associated with an attenuation of tumor necrosis factor alpha (TNF- $\alpha$ ) release in a mouse postischemic liver model (12). A correlation between the presence of MMP-2 in synovial fluid from patients with TMD and the degeneration of disc and articular cartilage has been reported (13). Moreover, a pioneer study demonstrated that early- and late-phase neuropathic pain that develops following nerve injury requires different MMPs (14).

MMP-9 shows rapid and transient upregulation in injured dorsal root ganglion (DRG) primary sensory neurons, which is consistent with early-phase neuropathic pain, whereas MMP-2 shows a delayed response in DRG satellite cells and spinal astrocytes, which is consistent with late-phase neuropathic pain (14). It is important to emphasize that satellite glial cells (SGCs) are peripheral glial cells and form a continuous layer around primary sensory neurons within DRG and trigeminal ganglia (TG). Furthermore, SGCs have been implicated in the regulation of neuronal homeostasis and neurotransmission in DRG and TG (15).

Although data from some studies support MMP involvement in inflammatory processes $(14,16)$, further evidence is required to clarify the physiological and pathological mechanisms of these extracellular proteolytic enzymes. The purpose of this investigation was to evaluate whether expression assayed by gelatin zymography and the level of gelatinolytic activity of MMP-2 and MMP-9 is altered in the trigeminal ganglion during different stages of temporomandibular inflammation. In addition, this study evaluated whether mechanical allodynia and orofacial hyperalgesia induced by the injection of CFA into the TMJ capsule could be altered by an MMP inhibitor (doxycycline, DOX). Moreover, this study measured TMJ inflammation using plasma extravasation in the periarticular tissue (Evans blue test) and infiltration of polymorphonuclear neutrophils into the synovial fluid (myeloperoxidase enzyme quantification).

\section{Material and Methods}

\section{Animals}

Experiments were performed with Wistar male rats ( $n=6-8$, for each experimental group) weighing 250-300 g, obtained from the animal facility of Universidade de São Paulo, Campus de Ribeirão Preto, Brazil. Animals were housed in a room with a controlled temperature $\left(24 \pm 1^{\circ} \mathrm{C}\right)$ and a 12-h light/dark cycle (lights on at 6:00 am) with food and water ad libitum. The experiments were carried out in compliance with the recommendations of SBNeC (the Brazilian Society of Neuroscience and Behavior) and with the approval of the Animal Care and Use Committee of Universidade de São Paulo, Campus Ribeirão Preto, SP, Brazil (Protocol \#09.1.371.53.0). All efforts were made to minimize animal suffering.

\section{Administration of CFA}

Initially, rats were anesthetized with an intramuscular injection of $75 \mathrm{mg} / \mathrm{kg} 10 \%$ ketamine and $10 \mathrm{mg} / \mathrm{kg} 4 \%$ xylazine followed by bilateral intraarticular administration of $50 \mu \mathrm{g}$ CFA (Mycobacterium tuberculosis) suspended in $50 \mu \mathrm{L}$ paraffin oil (Sigma, USA) or $0.9 \%$ saline solution (SAL). This dose was based on a previous report (17). A 26-G 1/2" needle attached to a 1-mL plastic syringe was used for the injection. To locate the TMJ for the injection, we palpated the zygomatic arch and the condyle. The needle was inserted immediately below the posteroinferior border of the zygomatic arch and advanced anteriorly to contact the edge of the posterolateral condyle (18).

\section{Removal of TG}

To quantify MMPs, a separate group of rats given CFA or $0.9 \%$ SAL into the TMJs as previously described were euthanized by an anesthesia overdose $(300 \mathrm{mg} / \mathrm{kg} \mathrm{10 \%}$ ketamine, plus $30 \mathrm{mg} / \mathrm{kg} 4 \%$ xilasine) at $1,3,7$, or 10 days after the beginning of the experiment. These time points were chosen based on a previous study (14). After 
euthanasia, the TG of each rat was removed bilaterally. The TGs were dissected and washed in $0.9 \%$ SAL to remove the blood contained in the sample, reducing potential interference in the results. After removal, all tissues were cut into pieces and the samples were used for extraction and quantification of proteins. Additionally, because some reports have demonstrated that low levels of MMPs can be expressed in the unstimulated brain (10), control groups without any TMJ manipulation were euthanized, and the TG were dissected and analyzed in all protocols used in this study.

\section{Quantification of Evans blue extravasation}

Extravasated Evans blue dye was quantitatively measured in the TMJ tissue by spectrophotometry. The different groups of rats were administered an iv injection of $25 \mathrm{mg} / \mathrm{kg}$ Evans blue dye dissolved in saline, $30 \mathrm{~min}$ before euthanasia, at $1,3,7$, or 10 days following the start of the experiments. Next, the periarticular tissue was dissected, weighed and fixed in $2 \mathrm{~mL}$ formaldehyde overnight. The supernatant $(100 \mu \mathrm{L})$ was extracted, and the absorbance at $630 \mathrm{~nm}$ was determined with a spectrophotometer. Dye concentration was assayed by comparison to a standard curve of known amounts of Evans blue dye in the extraction solution, which was assessed within the same assay. The quantity of Evans blue dye $(\mu \mathrm{g} / \mathrm{mL})$ in rat tissue exudate was then calculated (19).

\section{Analysis of myeloperoxidase activity}

Myeloperoxidase (MPO) is an enzyme found primarily in the azurophilic granules of neutrophils and has been used extensively as a biochemical marker of granulocyte infiltration in various tissues. Determining MPO activity has been described previously by Bradley et al. (20). In this study, the MPO assay was conducted in TMJ tissues at different time points following the start of the experiments $(1,3,7$, or 10 days) in the rat groups (Control, SAL and CFA). Briefly, 100-200 mg TMJ tissue was homogenized in $1 \mathrm{~mL}$ hexadecyltrimethylammonium bromide (HTAB) buffer for each $50 \mathrm{mg}$ tissue. The homogenate was centrifuged at $2600 \mathrm{~g}$ for $12 \mathrm{~min}$ at $4^{\circ} \mathrm{C}$. MPO activity in the resuspended pellet was assayed by measuring the change in absorbance at $450 \mathrm{~nm}$ using o-dianisidine dihydrochloride and $1 \%$ hydrogen peroxide. The results are reported as MPO U/ $\mu \mathrm{L}$ synovial fluid. A unit of MPO activity was defined as the conversion of $1 \mu \mathrm{mol}$ hydrogen peroxide to water in $1 \mathrm{~min}$ at $22^{\circ} \mathrm{C}$.

\section{Gelatin zymography of the TG}

Gelatin zymography is one of the most commonly employed methods for the quantification of MMP-2 and MMP-9. Under denaturing conditions, enzymes are separated by molecular weight using gel electrophoresis. Then, enzymes are refolded and the different molecular weight forms are visualized in zymograms. In the present study, gelatin zymography of MMP-2 and MMP-9 from the
TG was performed as previously described (21). Briefly, frozen ganglion samples $(0.08 \mathrm{~g})$ were homogenized in $300 \mu \mathrm{L}$ extraction buffer containing $10 \mathrm{mM} \mathrm{CaCl}_{2}, 50 \mathrm{mM}$ Tris- $\mathrm{HCl}, \mathrm{pH} 7.4,1 \mathrm{mM}$ Phe (1,10 ortho-phenanthroline), $1 \mathrm{M}$ phenylmethanesulfonylfluoride (PMSF) and $1 \mathrm{mM} \mathrm{N}$ ethylmaleimide. These samples were placed in the refrigerator for $20 \mathrm{~h}$ on ice to extract the proteins. The samples were then centrifuged at $3000 \mathrm{~g}$ for $15 \mathrm{~min}$. The protein content was measured using the Bradford method (22). The samples were then diluted in sample buffer $(2 \%$ SDS, $125 \mathrm{mM}$ Tris- $\mathrm{HCl}, \mathrm{pH} 6.8,10 \%$ glycerol, and $0.001 \%$ bromophenol blue) and subjected to electrophoresis on $12 \%$ SDS-PAGE co-polymerized with $1 \%$ gelatin as the substrate. After electrophoresis was completed, the gel was incubated for $1 \mathrm{~h}$ at room temperature in a $2 \%$ Triton $\mathrm{X}-100$ solution, followed by incubation at $37^{\circ} \mathrm{C}$ for $16 \mathrm{~h}$ in Tris- $\mathrm{HCl}$ buffer, $\mathrm{pH} 7.4$, containing $10 \mathrm{mM} \mathrm{CaCl}_{2}$. The gels were stained with $0.05 \%$ Coomassie brilliant blue G-250 and then destained with 30\% methanol and $10 \%$ acetic acid. Gelatinolytic activities were detected as unstained bands against the background of Coomassie blue-stained gelatin. Enzyme activity was determined by densitometry using a Kodak Electrophoresis Documentation and Analysis System (EDAS 290; Kodak, USA). Gelatinolytic activities were normalized against an internal standard (fetal bovine serum) to allow intergel analyses and comparisons. Based on a previous study (23), the bands at 75, 72, and $64 \mathrm{kDa}$ identified MMP-2, and MMP9 was identified by a $92-k D a$ band.

\section{In situ zymography and immunofluorescence assays of gelatinolytic activity and MMP-2 and MMP-9 expression}

In situ MMP activity was measured in frozen TGs (5 per group) using DQ Gelatin (E12055, Molecular Probes, USA) as a fluorogenic substrate. Briefly, TG samples were embedded in Tissue Tek and cut into $5-\mu \mathrm{m}$ sections with a cryostat. Sample sections were incubated with $1.0 \mathrm{mg} / \mathrm{mL} \mathrm{DQ}$ gelatin in Tris- $\mathrm{CaCl}_{2}$ buffer $(50 \mathrm{mM}$ Tris, $10 \mathrm{mM} \mathrm{CaCl}_{2}, 1 \mathrm{mM} \mathrm{ZnCl}_{2}$ ) in dark, humidified chambers for $1 \mathrm{~h}$. The sections were examined with fluorescence microscopy (Leica Imaging Systems Ltd., England) and the image was captured at a magnification of $400 \times$. Negative control sections were incubated in the same way as described above, but without DQ gelatin. Some sections were incubated with a metalloproteinase inhibitor, Phe, or a serine protease inhibitor, PMSF (Sigma Chemicals, USA), at $2 \mathrm{mM}$, or with both inhibitors. Proteolytic activity was detected as bright green fluorescence, which indicated substrate breakdown, and was evaluated using the ImageJ Program (National Institutes of Health, USA). To evaluate MMP-2 and MMP-9 expression, 5- $\mu \mathrm{m}$ tissue sections were incubated with mouse anti-MMP-2 monoclonal antibody or mouse antiMMP-9 monoclonal antibody (MAB3308 or MAB3309, 1:1000 dilution; Chemicon, USA) for $1 \mathrm{~h}$ in a dark 
humidified chamber. Red fluorescence was visualized by adding a rhodamine-conjugated anti-mouse secondary antibody (AP160P, 1:200; Chemicon) for $1 \mathrm{~h}$. DAPI (4',6diamidino-2-phenylindole) was applied for $3 \mathrm{~min}$, and specimens were washed with phosphate-buffered saline (PBS) and assayed by fluorescence microscopy to identify cell nuclei. To confirm the specificity of antibodies, the primary antibody was omitted and substituted by PBS with $1 \%$ BSA. Rhodamine did not bind nonspecifically to these control tissue sections (24). MMP-2 and MMP-9 expression were detected as bright red fluorescence, whereas DAPI was detected as blue fluorescence; all images were evaluated using the Image $\mathrm{J}$ software.

\section{Mechanical orofacial sensitivity}

To assess mechanical orofacial sensitivity before (control period) and 10 days after the bilateral administration of CFA (CFA group) or $0.9 \%$ saline (SAL group) into the TMJ, we evaluated the head withdrawal reflex during the application of the mechanical stimuli. To measure the head withdrawal reflex, rats were placed in the testing chamber for a minimum 30 -min adaptation period. Progressive, increasing forces from the filament of an electronic von Frey anesthesiometer (Insight Instruments, Brazil) were applied to the TMJ region until the head was withdrawn. The applied force was recorded. The head withdrawal threshold of each rat was calculated as means \pm SE of 3 values obtained in each session (control period and 10 days later). All experiments were carried out in a quiet room between 8:30 and 10:30 am to avoid diurnal variations.

To assess the role of the MMPs on mechanical orofacial sensitivity, separate groups of rats were administered SAL or CFA injections and DOX (30 mg $\mathrm{kg}^{-1} \cdot \mathrm{day}^{-1}$; $\mathrm{SAL}+\mathrm{DOX}, \mathrm{CFA}+\mathrm{DOX}$ ) or vehicle (distilled water; $S A L+V E H I, C F A+V E H I)$ by gavage for 10 days (21), beginning on the day of the SAL or CFA injection. These groups were subjected to mechanical sensitivity evaluations as previously described.

\section{Nociceptive orofacial sensitivity}

To evaluate the nociceptive response of rats to a sharp stimulus to the orofacial region, we performed an orofacial formalin test in three experimental groups. The first and second groups received bilateral injections of $0.9 \%$ saline into the TMJ. The third group received bilateral injections of CFA into the TMJs as previously described. Ten days after initial injection, group one received a subcutaneous injection of $0.9 \%$ saline $(50 \mu \mathrm{L})$ into the vibrissa pad $(S A L+S A L)$. The remaining two groups received a subcutaneous injection of $2 \%$ formalin $(50 \mu \mathrm{L})$ in the same area (SAL+FORM and CFA+FORM). For the administration of saline or formalin solutions, the rats were allowed to adapt to a testing chamber for $20 \mathrm{~min}$. The experimental room had little human activity and a controlled temperature of $25 \pm 1^{\circ} \mathrm{C}$. The animals were removed from the box and a volume of
$50 \mu \mathrm{L} 2 \%$ formalin or $0.9 \%$ saline was injected subcutaneously into the orofacial region between the nose and the upper lip. A 26-G 1/2" needle attached to a 1-mL plastic syringe was used for the injections. Injections were performed as quickly as possible to avoid prolonged handling that could interfere with the results of this study. Immediately after the injection, rats were returned to the testing chamber, and the number of seconds they spent rubbing the ipsilateral face was recorded for 15 intervals of 3 min each. According to Grabow and Dougherty (25), the orofacial formalin test can be characterized by two phases. Phase 1 is the first interval of vibrissal rubbing (0 to $3 \mathrm{~min}$ ) and phase 2 is defined as the period of vibrissal rubbing between interval 5 and interval 15 (i.e., from min 15 to 45 of the test period). In general, the peak of the vibrissal rubbing in phase 2 was observed during interval 7 ( $\min 21$ to 24 ) and diminished before interval 15 ( $\min 43$ to 45 ).

To evaluate the involvement of the MMPs in nociceptive orofacial sensitivity, separate groups of rats were given SAL or CFA injections into the TMJ and received DOX (30 $\left.\mathrm{mg} \cdot \mathrm{kg}^{-1} \cdot \mathrm{day}^{-1} ; \mathrm{SAL}+\mathrm{DOX}, \mathrm{CFA}+\mathrm{DOX}\right)$ or vehicle (distilled water; SAL + VEHI, CFA + VEHI) by gavage for 10 days (21) beginning on the day of the TMJ injection. These animals were submitted to the formalin orofacial test at the end of the treatment (day 10).

\section{Statistical analysis}

Statistical analysis of the two inflammation markers (Evans blue absorbance and MPO activity) was performed by two-way analysis of variance (ANOVA) followed by the Newman-Keuls post hoc test, with the level of significance set at $\mathrm{P}<0.05$.

The images of the MMP zymograms were quantified using the ImageJ program, and the results are reported as means \pm SE of arbitrary units. For all protocols, betweengroup comparisons were assessed by one-way or two-way ANOVA followed by the Newman-Keuls post hoc test. A probability value of $\mathrm{P}<0.05$ was considered to be significant.

Mechanical sensitivity results are reported as means \pm SE of the threshold $(\mathrm{g})$ of the head withdrawal reflex testing. To analyze the results of the nociceptive sensitivity test, the duration in seconds that animals spent rubbing the vibrissal pad during the fifteen 3-min intervals was reported as means \pm SE for each interval. For both protocols, comparisons between the groups were assessed by one-way or two-way ANOVA followed by a Newman-Keuls test. A probability value of $\mathrm{P}<0.05$ was considered to be significant.

\section{Results}

\section{Quantification of plasma extravasation and myeloperoxidase activity in TMJs}

The administration of CFA bilaterally in the TMJ region promoted greater plasma extravasation in the area compared with groups without inflammation (SAL and 
Control, Figure 1A). Two-way ANOVA showed a significant treatment effect $\left(F_{2,87}=78.77, P<0.001\right)$ and an interaction between treatment and time after CFA-induced inflammation $\left(1,3,7\right.$, and 10 days; $F_{6,87}=4.602$, $\mathrm{P}<0.001)$. The Newman-Keuls post hoc test revealed a significant difference $(P<0.05)$ in extravasated plasma in the CFA group compared with the SAL and Control groups for all the periods analyzed (Figure 1A).

The MPO activity in the TMJs is shown in Figure 1B. The administration of CFA into the TMJ promoted an increase in MPO levels in the periarticular tissue (Figure 1B). Two-way ANOVA demonstrated a significant treatment effect $\left(F_{2,87}=600.49, P<0.001\right)$ and an interaction between treatment and time after CFA-induced inflammation $\left(1,3,7\right.$, and 10 days; $\left.F_{6,87}=131.49, P<0.001\right)$. MPO levels were elevated in the CFA group compared with the Control and SAL groups for all periods analyzed (Newman-Keuls post hoc test, $\mathrm{P}<0.05$ ). In addition, this increase was more evident on days 1 and 3 following the start of the experiments (Figure 1B). Furthermore, no differences were observed between the Control and SAL groups in any of the experimental periods.

\section{Quantification of plasma extravasation and MPO activity in TMJs after treatment with a nonspecific MMP inhibitor}

The systemic administration of DOX for 10 days promoted a reduction in plasma extravasation in the TMJ region of animals given CFA injections, as shown in Figure 2A. One-way ANOVA showed a significant treatment effect $\left(F_{1,23}=3.84, P<0.05\right)$. The Newman-Keuls post hoc test revealed a difference $(P<0.05)$ in extravasated plasma in the CFA group compared with the other groups analyzed.

MPO activity in the TMJs after DOX treatment is presented in Figure 2B. One-way ANOVA followed by the Newman-Keuls post hoc test showed a difference in the CFA + VEHI group compared with the groups given a nonspecific MMP inhibitor or the Control group $\left(F_{1,23}=1.81, P<0.001\right.$, ANOVA $)$.

\section{Quantification of MMP-2 and MMP-9 in the TG by zymography}

The results of the MMP quantifications revealed that MMP-2 and MMP-9 levels were greater in rats that received CFA than in the SAL and Control groups in different periods following the start of the experiments (Figure 3A-F). Representative SDS-PAGE gelatin zymograms of the different periods analyzed are presented in Figure $3 \mathrm{~A}$ and $\mathrm{B}$.

Two-way ANOVA showed significant differences in MMP-9 levels for time $\left(F_{3,54}=15.618, P<0.001\right)$, treatment $\left(F_{1,54}=18.961, P<0.001\right)$, and an interaction between time and treatment $\left(F_{3,54}=3.715, P<0.01\right)$. The Newman-Keuls post hoc test revealed a significant increase $(\mathrm{P}<0.05)$ in MMP-9 in the CFA groups on days 1 and 3 following the onset of inflammation compared with the Control and SAL groups (Figure 3C). Statistical analyses revealed no significant differences in the levels of MMP-9 on days 7 and 10 (Figure 3C).

Regarding MMP-2, two-way ANOVA applied to the 75kDa MMP-2 data showed a significant time effect $\left(F_{3,67}=16.312, P<0.001\right)$ and treatment $\left(F_{1,67}=40.352\right.$, $\mathrm{P}<0.001$ ), but did not demonstrate an interaction between time and treatment. The post hoc Newman-Keuls test revealed a significant difference in $75-\mathrm{kDa} M M P-2$ in the CFA group compared with the Control and SAL groups (Figure 3D). Significant effects were shown (two-way ANOVA) for time $\left(F_{3,62}=88.87, P<0.001\right)$ and treatment $\left(F_{1,62}=48.57, P<0.001\right)$, together with an interaction between time and treatment $\left(\mathrm{F}_{3,62}=6.259, \mathrm{P}<0.001\right)$ for the $72-k D a$ MMP-2 band. The CFA-treated rats exhibited an increase in this band on days 1, 3, 7, and 10 following the initial injection compared with the Control and SAL groups on the same day $(\mathrm{P}<0.05$, Newman-Keuls post hoc test; Figure 3E). Statistical analysis of the $64-\mathrm{kDa}$ MMP-2 data revealed significant effects for time $\left(F_{3,67}=7.00, P<0.001\right)$ and treatment $\left(F_{1,67}=94.71\right.$, $\mathrm{P}<0.001$ ), and an interaction between time and treatment $\left(F_{3,67}=3.409, P<0.05\right)$. The Newman-Keuls post hoc test revealed a significant increase in the level of $64-\mathrm{kDa}$ MMP-2 expression in the TG in the CFA groups $(\mathrm{P}<0.05$, Newman-Keuls test) throughout the experimental period when compared with Control and SAL groups (Figure 3F).

\section{MMP activity and MMP-2 and MMP-9 expression} during the development of inflammation in the TMJ

Representative immunofluorescence photomicrographs showing total gelatinolytic activity, and MMP-2
A

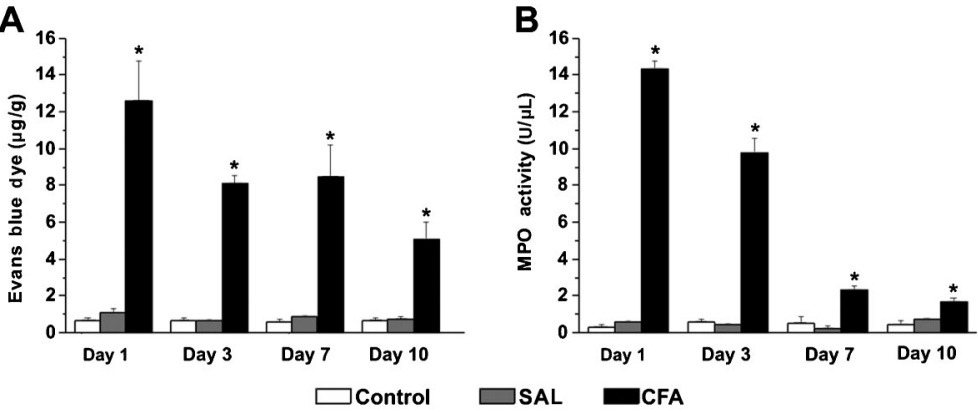

Figure 1. Evaluation of inflammation based on Evans blue dye in temporomandibular joint tissues $(A)$ and myeloperoxidase (MPO) activity in the synovial fluid $(B)$ of the rats after different periods following administration of complete Freund's adjuvant (CFA) or saline (SAL) or without any application (Control). Data are reported as means \pm SE ( $n=6$ per group). ${ }^{*} \mathrm{P}<0.05$, compared with Control and $\mathrm{SAL}$ groups (ANOVA and Newman-Keuls test). 


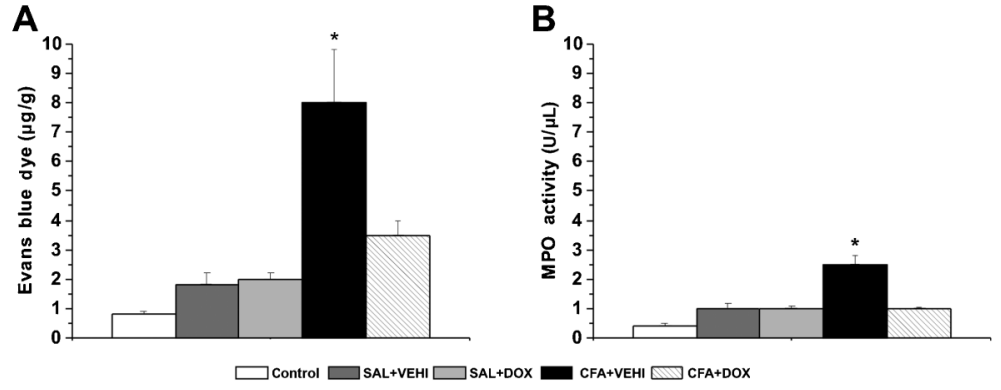

Figure 2. Evaluation of inflammation based on Evans blue dye in temporomandibular joint tissues $(A)$ and myeloperoxidase (MPO) activity in the synovial fluid $(B)$ of the rats after administration of doxycycline (SAL+DOX and CFA+DOX), vehicle of DOX (SAL + VEHI and CFA + VEHI) systemically for 10 days or without any treatment (Control). CFA: complete Freund's adjuvant; SAL: saline. Data are reported as means $\pm S E\left(n=6\right.$ per group). ${ }^{*} P<0.05$, compared with Control, SAL+VEHI, SAL + DOX and CFA+DOX groups (ANOVA and NewmanKeuls test). and MMP-9 expression in TG tissue are presented in Figure $4 \mathrm{~A}$ and $\mathrm{B}$. Two-way ANOVA of the total gelatinolytic activity in TG showed significant effects for time $\left(F_{3,63}=412.4, P<0.001\right)$ and treatment $\left(F_{1,63}=499.3\right.$, $\mathrm{P}<0.001)$, and an interaction between time and treatment
$\left(F_{3,63}=4.123, P<0.001\right)$. The Newman-Keuls post hoc test demonstrated that the total gelatinolytic activity in rats with temporomandibular inflammation (CFA group) at 1, 3, 7 , and 10 days was higher than the activity in the Control and SAL groups (Figure 4C) for the same period
A

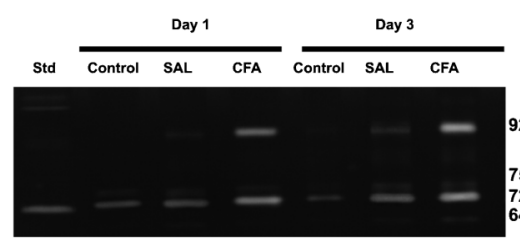

C

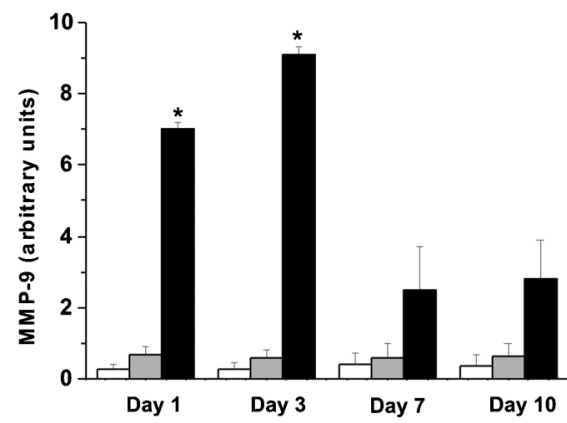

E

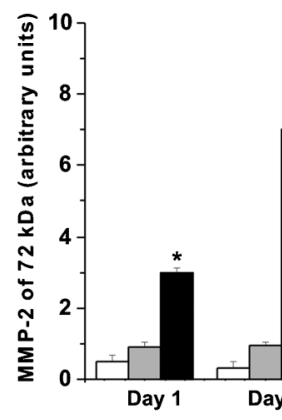

B

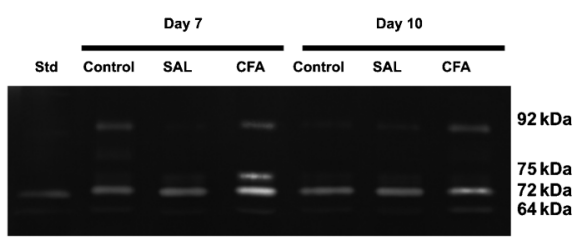

D

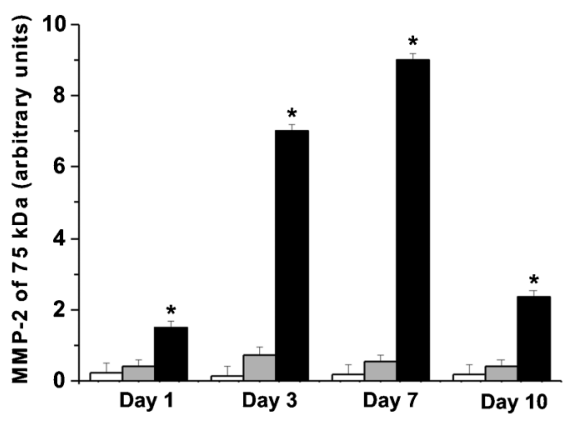

$\mathbf{F}$

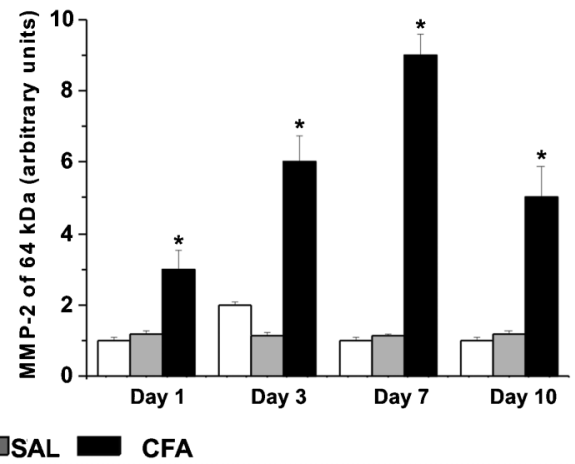

Figure 3. Representative SDS-PAGE gelatin zymograms of trigeminal ganglion extracts (TGs) 1,3 , 7 , and 10 days following complete Freund's adjuvant-induced inflammation (CFA), administration of saline (SAL) or without any treatment (Control, Panels $A$ and $B$ ). Std: internal standard. Molecular weights of MMP-9 band $(92 \mathrm{kDa})$ and MMP-2 bands $(75,72$, and $64 \mathrm{kDa})$ were identified after electrophoresis on $12 \%$ SDS-PAGE. Gels were loaded with $40 \mu \mathrm{g}$ protein in each lane. Panels $C, D, E$, and $F$ show the values for the 92-, 75-, 72-, and 64-kDa molecular weights, respectively, in the TGs. Data are reported as means $\pm S E\left(n=8\right.$ per group). ${ }^{*} P<0.05$, compared with the respective SAL and Control groups (Newman-Keuls test). 
$(P<0.05)$. No differences were observed between the Control and SAL groups.

Regarding MMP expression, two-way ANOVA applied to the MMP-2 expression data showed significant effects for time $\left(F_{2,62}=88.87, P<0.001\right)$ and treatment $\left(F_{1,62}=48.57, P<0.001\right)$, and an interaction between time and treatment $\left(F_{3,62}=6.25, P<0.001\right)$. The MMP-2 levels in the CFA group were increased compared with the Control and SAL groups on days 7 and $10(\mathrm{P}<0.05$, Newman-Keuls post hoc test; Figure 4D).

Regarding MMP-9 expression, significant effects for time $\left(F_{3,67}=7.003, P<0.001\right)$, and treatment $\left(F_{1,67}=94.717, P<0.001\right)$, and an interaction between time and treatment $\left(F_{3,67}=3.409, P<0.001\right)$ were shown by two-way ANOVA. The Newman-Keuls post hoc test revealed a significant increase $(P<0.05)$ in MMP-9 expression in the CFA groups on days 1 and 3 following the onset of inflammation compared with the Control and SAL groups (Figure 4E).

\section{Evaluation of mechanical sensitivity in rats with persistent TMJ inflammation}

The results show that bilateral CFA administration to the TMJ reduced the mechanical threshold of the withdrawal reflex (Figure 5A). No significant decrease in the mechanical threshold was observed 10 days after the injection of SAL into the TMJ. The CFA group showed a significant difference $\left(\mathrm{F}_{1,11}=7.59, \mathrm{P}<0.05\right.$, ANOVA, $\mathrm{P}<0.05$, Newman-Keuls) between the initial period before treatment and 10 days after the injection (Figure 5A). Treatment with DOX abolished the reduction in the mechanical orofacial threshold following the injection of CFA. Statistical analysis did not show a difference between the SAL + VEHI and SAL + DOX groups on day 10 when compared to the control period $\left(F_{1,15}=0.983\right.$, $\mathrm{P}>0.05$, ANOVA; Figure 5B). In contrast, the CFA+VEH and CFA+DOX groups showed a significant difference $\left(F_{1,15}=17.308, P<0.05\right.$, ANOVA $)$. The Newman-Keuls post hoc test $(\mathrm{P}<0.05)$ demonstrated that treatment with

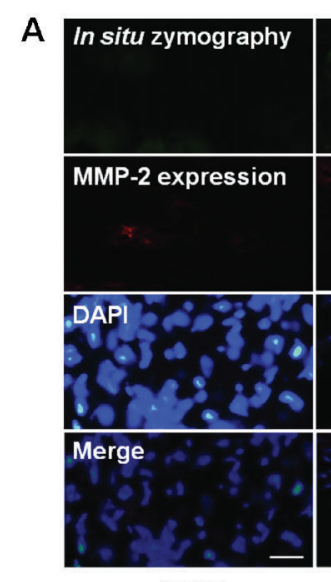

Control

C

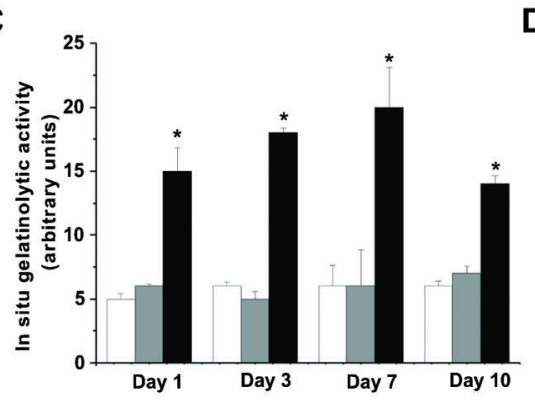

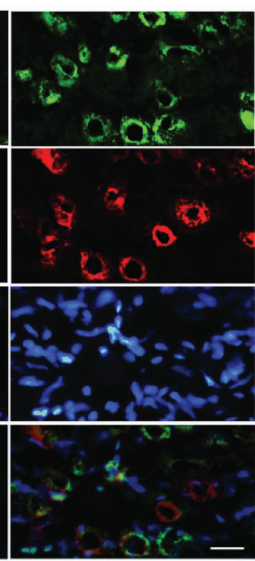

CFA
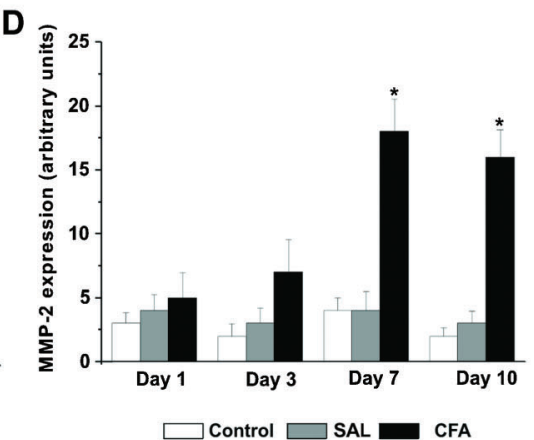
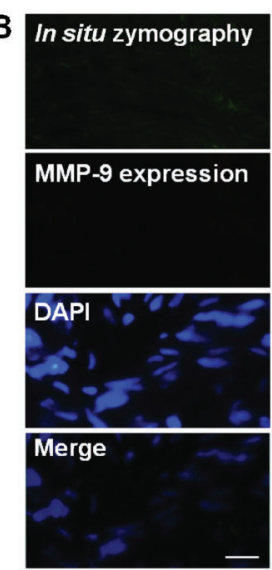

Control

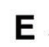

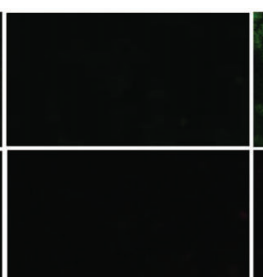
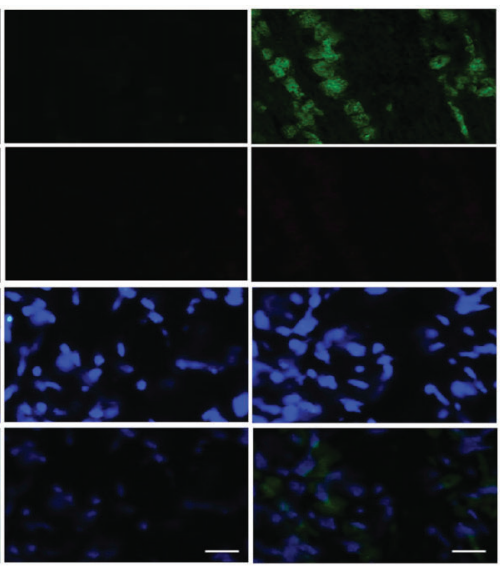

SAL

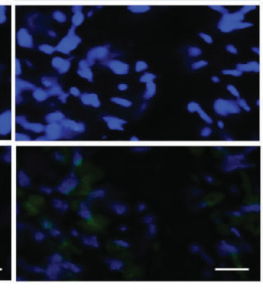

CFA

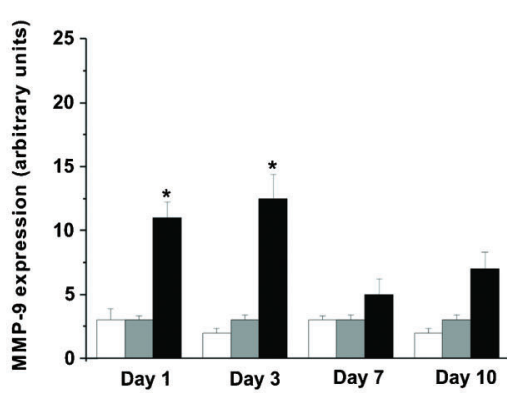

Figure 4. In situ gelatinolytic activity, MMP-2 and MMP-9 expression in the trigeminal ganglion (TG) from rats. Pane/s $A$ and $B$, representative photomicrographs of gelatinolytic activity on day 7 (in situ zymograph), MMP-2 or MMP-9 levels, DAPI staining, and their co-localization in the TGs (Merge). Magnification bars: $100 \mu \mathrm{m}$. Panel $C$, mean gelatinolytic activity in TGs in each group assessed by the measurement of bright green fluorescence ( $n=6$ per group). Panel $D$, mean MMP-2 levels in the TGs in each group assessed by bright red fluorescence ( $n=6$ per group). Panel $E$, mean MMP-9 levels in the TGs in each group assessed by bright red fluorescence ( $n=6$ per group). CFA: complete Freund's adjuvant; $S A L$ : saline. Data are reported as means $\pm S E$. * $P 0.05, C F A$ group $v s$ SAL and Control groups (Newman-Keuls test). 
DOX blocked the decline in mechanical orofacial threshold induced by CFA administration to the TMJs (Figure $5 \mathrm{C})$.

\section{Evaluation of nociceptive sensitivity in rats with persistent TMJ inflammation}

The results show that, when compared with the SAL + FORM group, animals in the CFA + FORM group exhibited an increase in the frequency of orofacial area rubbing after formalin administration at both the beginning (phase 1) and end (phase 2) of the test (Figure $5 D)$. In the $S A L+S A L$ group, nociceptive behavior in the orofacial area was minimal and exhibited a stable pattern throughout the duration of the test (Figure 5E). The mean duration of orofacial rubbing was $1.1 \pm 0.7 \mathrm{~s}$ during phase 1 , and no orofacial rubbing was observed during the 21-24-min interval. In the SAL +FORM group, the mean duration of nociceptive behavior was $19.8 \pm 4$ s during phase 1 ( 0 to $3 \mathrm{~min}$ ) and was maintained at a high level throughout the duration of phase 2. During the 21-24-min interval, the mean duration of nociceptive behavior was $42.8 \pm 7.4 \mathrm{~s}$ (Figure $5 \mathrm{D}$ ). The CFA + FORM group showed a higher nociceptive behavior score; the mean time of orofacial rubbing was $28.9 \pm 5.7 \mathrm{~s}$ in phase 1 and $109.8 \pm 10 \mathrm{~s}$ in the 2124-min interval (Figure 5D). A two-way ANOVA for all experimental groups revealed significant effects of time $\left(F_{14,299}=12.354, \quad P<0.001\right)$ and treatment $\left(F_{2,299}=214.824, P<0.001\right)$, and there was an interaction between time and treatment $\left(F_{28,299}=4.935\right.$, $\mathrm{P}<0.001)$. The Newman-Keuls post hoc test showed that the mean duration of nociceptive behavior exhibited by the SAL +FORM and CFA+FORM groups during both phases 1 and 2 was significantly different $(P<0.05)$ compared to that of the $S A L+S A L$ group (Figure 5D). In addition, orofacial rubbing in the CFA + FORM group was significantly higher (Newman-Keuls, $\mathrm{P}<0.05$ ) than in the $\mathrm{SAL}+\mathrm{FORM}$ group during phase 2 (Figure 5D).

Treatment with DOX, a nonspecific MMP inhibitor, blocked the increase in nociceptive orofacial behavior induced by CFA injection (Figure $5 E$ and $F$ ). The two-way ANOVA revealed significant effects of time $\left(F_{14,359}=9.859, P<0.001\right)$ and treatment $\left(F_{2,359}=\right.$ 38.907, $\mathrm{P}<0.001)$, and found an interaction between time and treatment $\left(F_{42,359}=2.191, P<0.001\right)$. The NewmanKeuls test demonstrated that the mean rubbing duration in the CFA + VEHI group differed from that of the CFA+DOX group $(P<0.05$, Figure $5 F)$ in phase 2 of the formalin orofacial test. Moreover, the duration of nociceptive orofacial behavior in the CFA + VEHI group was significantly longer than that in the CFA+DOX, $S A L+V E H I$ and SAL + DOX groups, particularly during phase 2. The peak nociceptive behavior occurred during the $27-30-\mathrm{min}$ interval of the $45-\mathrm{min}$ test period (Figure 5F).

\section{Discussion}

Several studies have reported that inflammation processes in the TMJ cause the production of proinflammatory substances, including tachykinins such as substance $P$ and calcitonin gene-related peptides (CGRPs) (17). In addition, clinical analysis has found that TMD patients have high levels of the cytokine interleukin 1-beta (IL-1 $\beta$ ) in the synovial fluid (26). Our results corroborate a study by Spears et al. (17), in which the development of CFA inflammation of the TMJ was followed by variable increases in the concentration of inflammatory mediators in both the TG and TMJ tissue. In particular, in TMJ tissue, CFA inflammation promoted an increase of CGRPs, nerve growth factor (NGF), IL-1 $\beta$, and TNF- $\alpha$ at 2 days, and they remained elevated throughout the 6-week experimental period. Similarly, in the TG, the inflammatory mediators CGRP and NGF were elevated 2 days, and 2 and 4 weeks after CFA injection (17). In addition, during an inflammatory process, increased or dysregulated levels of numerous MMPs were observed (11). Although MMP inhibitors have been used as anti-inflammatory drugs (27), the specific role of individual MMPs needs to be clarified.

One report hypothesized that neuropathic pain and neuroinflammation share similar mechanisms (14). The authors showed that in cultured DRG neurons, TNF- $\alpha$ and IL-1 $\beta$ produced following tissue injury promoted an increase in both the expression and release of MMP-9. Furthermore, a TNF- $\alpha$ inhibitor suppressed MMP-9 upregulation induced by spinal nerve ligation. Thus, it is reasonable to hypothesize that TMJ inflammation induced by bilateral administration of CFA promotes an increase in inflammatory mediators (17), which further promote the upregulation of MMPs (MMP-9 and MMP-2) in the TG.

The results obtained here demonstrate that bilateral administration of CFA in the region of the TMJs promoted persistent inflammation, demonstrated by an increase in both temporomandibular plasma extravasation and MPO activity in the synovial fluid (Figure 1). In addition, the data showed increased expression of MMP-9 and MMP-2 in the TG during different periods of the experiment (Figure 4). Specifically, MMP-9 expression was greater in the early phase of the inflammatory process (days 1 and 3 following CFA administration into the TMJs), and MMP-2 expression was greater in the CFA group on days 7 and 10 following the start of the experiments. It is important to note that MMP-2 is constitutively expressed in many tissues whereas MMP-9 is highly inducible (16). So, it is possible that the MMP-2 shown in Figure 3 is the constitutive form of this gelactinase. On the other hand, the results in Figure 3 show the in situ gelatinolytic activity of the MMPs, and MMP-2 and MMP-9 expression during the development of inflammation in the temporomandibular joint. So, in Figure 3, we have evidence that MMP-2 expression was greater in the CFA group on days 7 and 

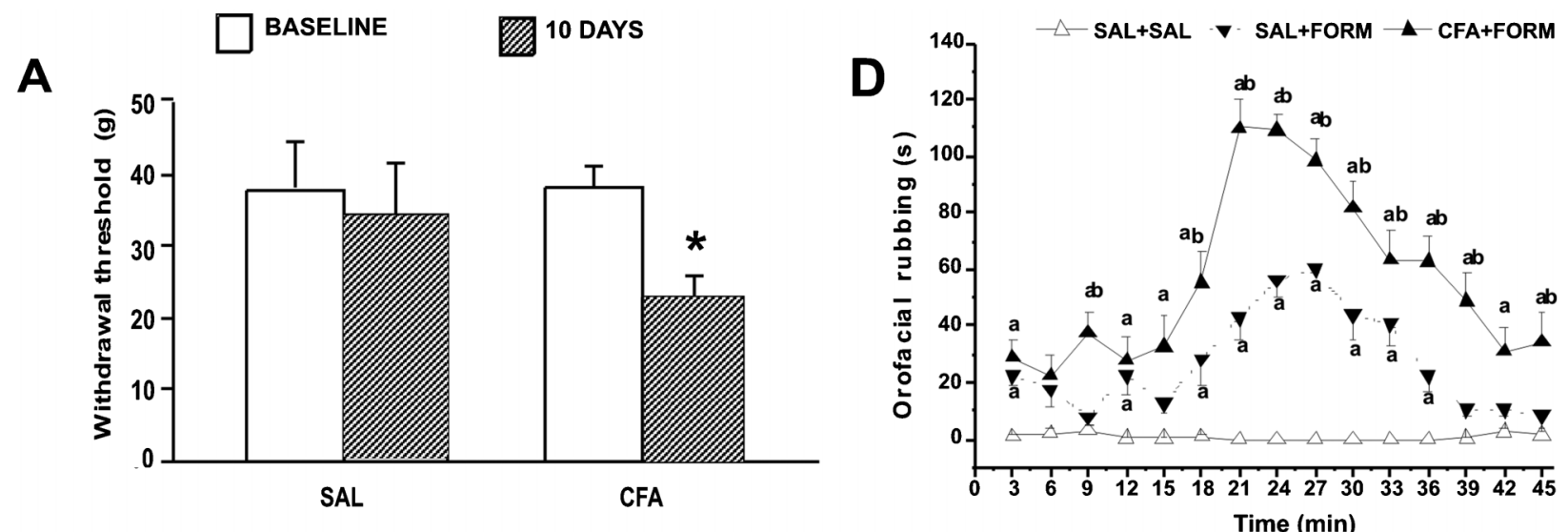

B

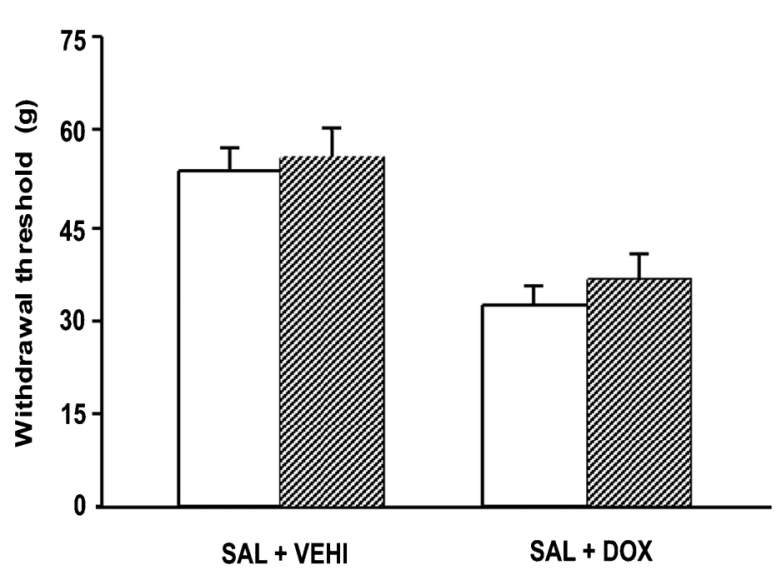

$\mathbf{E}$

Time (min)

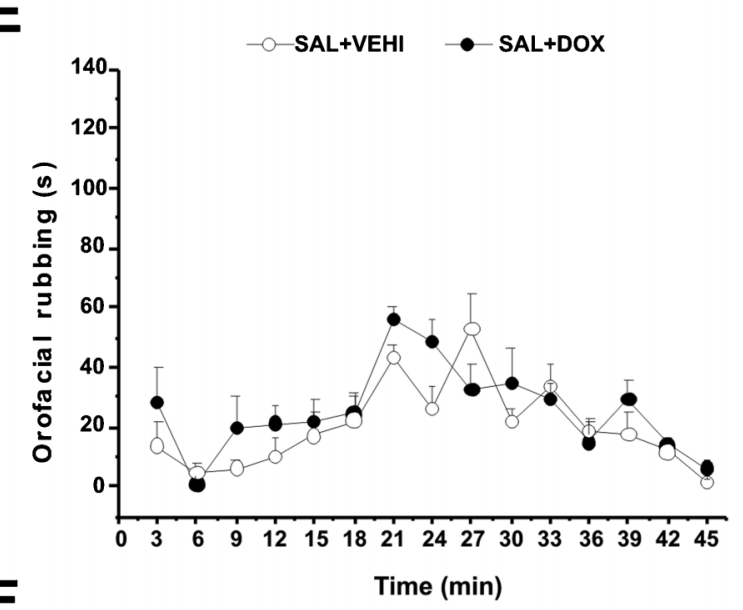

C
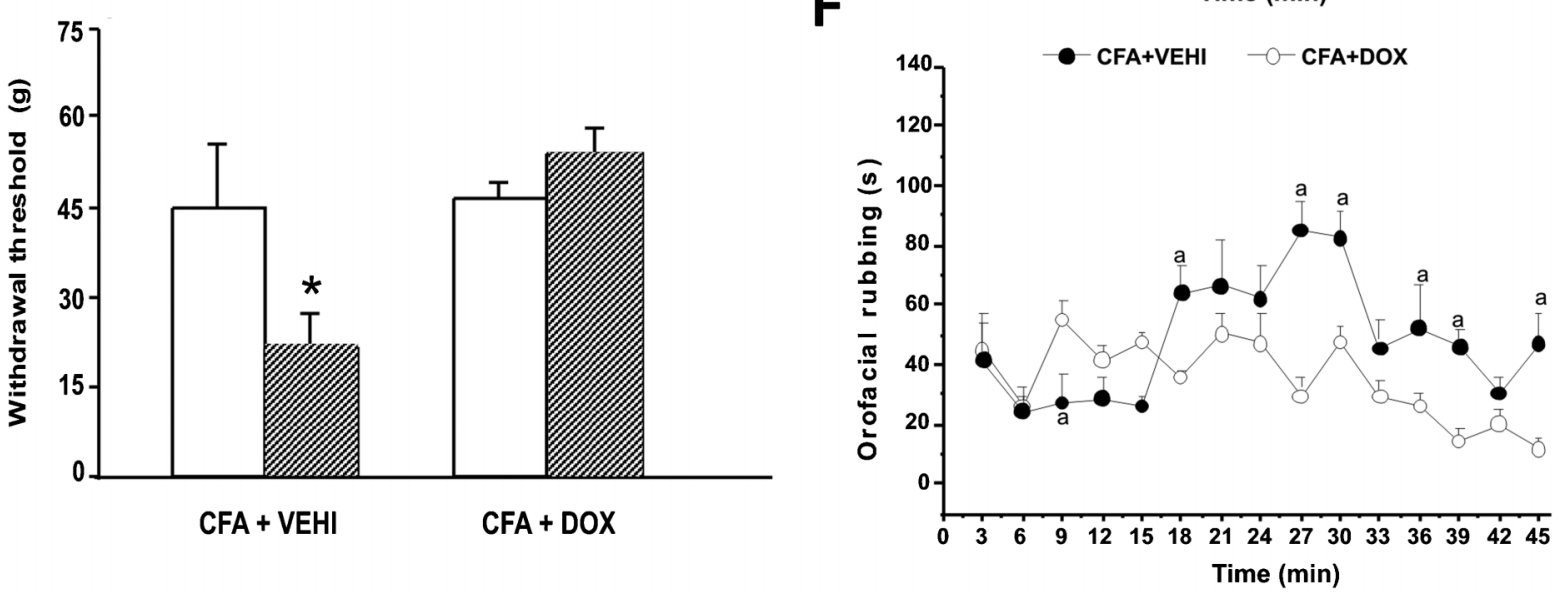

Figure 5. Mechanical and nociceptive sensitivity. Panels $A, B$, and $C$, mechanical sensitivity in the control period (baseline) and 10 days after treatment. $A$, Mechanical threshold $(\mathrm{g})$ of the withdrawal reflex of rats that received $0.9 \%$ saline (SAL) or complete Freund's adjuvant (CFA) injected into temporomandibular joints (TMJs). B, Mechanical threshold ( $\mathrm{g}$ ) of the withdrawal reflex of rats that received SAL injected into TMJs and were treated with doxycycline (DOX, $30 \mathrm{mg} \cdot \mathrm{kg}^{-1} \cdot$ day $^{-1}$ ) or vehicle (VEHI, distilled water) for 10 days by gavage. $C$, Mechanical threshold $(\mathrm{g})$ of the withdrawal reflex of rats that received CFA injected into TMJs and were treated with DOX or VEHI for 10 days. $D$, Orofacial rubbing (s) of rats that received SAL or CFA injected into TMJs and 10 days later were submitted to administration of SAL or $2 \%$ formalin (FORM) in the vibrissal pad. E, Orofacial rubbing (s) of rats that received SAL injected into TMJs and were treated with DOX or VEHI and submitted to injection of FORM in the vibrissal pad. $F$, Orofacial rubbing (s) of rats that received CFA injected into TMJs and were treated with DOX or VEHI and 10 days later were submitted to injection of FORM in the vibrissal pad. Data are reported as means $\pm \mathrm{SE}$. ${ }^{\mathrm{a}} \mathrm{P}<0.05$, compared with $\mathrm{SAL}+\mathrm{SAL}$ or $\mathrm{CFA}+\mathrm{DOX}$ (Newman-Keuls test). ${ }^{\mathrm{b}} \mathrm{P}<0.05$, compared with SAL + FORM (Newman-Keuls test). 
10, whereas MMP-9 expression was greater on days 1 and 3 .

The present results also showed that bilateral administration of CFA into the TMJ reduced the mechanical orofacial threshold of the withdrawal reflex after the application of an innocuous mechanical stimulus, and promoted an increase in orofacial rubbing during the formalin test (Figure 5A-F). The results also demonstrate that treatment with a nonspecific MMP inhibitor, DOX, reduced mechanical allodynia and orofacial hyperalgesia, thus reinforcing the role of the MMPs in mechanical and nociceptive orofacial sensitivity. Additionally, we observed that 10 days of treatment with an MMP inhibitor diminished both Evan's blue extravasation and MPO activity (Figure $2 \mathrm{~A}$ and $\mathrm{B}$ ), suggesting a reduction of inflammatory processes.

According to the literature, hyperalgesia is promoted by the actions of CFA in the TMJ and promotes nociceptive behaviors, such as rubbing of the orofacial region, self-care behavior, and attention to the body surface (28). Congruent with the present results, a previous report also demonstrated that the head withdrawal threshold significantly decreased 3 days after CFA was administered into the TMJs (7).

Considering the sensory neural pathways, sensory ganglion neurons occupy a unique position in these ascending pathways, and represent the first location in the nervous system where sensations are generated, particularly nociceptive sensitivity. In addition, there is increasing evidence that these ganglion cells contribute to the establishment of persistent, chronic pain (29). In fact, among the variety of substances released by activated immune and glial cells, proinflammatory cytokines (TNF$\alpha, I L-1, I L-6)$ appear to be of particular importance in neuronal hyperexcitability (29). The satellite glial cells in DRG and TG have gained attention in recent years, particularly for their involvement in pain facilitation $(30,31)$. These SGCs act as a mechanical barrier to neurons of the DRG and TG (15), participate in neurotransmitter reuptake mechanisms and can exert fine control of the neuronal microenvironment (32). Further evidence has shown that SGCs are involved in the morphological and biochemical changes that occur following peripheral nerve injury and inflammation (31). Over time, strong expression of MMP-9 occurs in neural tissue, including neurons and glial cells (33). Moreover, while MMP-2 is considered a constitutive gelatinase, MMP-9 shows highly inducible and transient expression, with one major input for its expression, the tissue inflammatory response (16). The main physiological function of the gelatinase, MMP-9, is maintaining synaptic plasticity in neural tissue; MMP-2 is correlated with tissue development and regeneration (34). Importantly, MMPs produced by ganglion cells in response to various stimuli, such as an inflammatory process, can be transported from the neuronal soma to the periphery, promoting their effects both within the ganglion and peripherally (16).

Taken together, our findings suggest that MMP-9 and MMP-2 upregulation actively participates in the inflammation process induced by CFA in the TMJ. Moreover, MMP9 and MMP-2 show expression in the establishment of the inflammatory process, since MMP-9 upregulation occurred immediately following the onset of inflammation induced by CFA in the TMJs, while the increase in MMP-2 (72 kDa) was detected in greater quantities on experimental day 7. Previous reports have shown that MMP-9 and MMP-2 are significantly involved in persistent pain $(11,14,35)$. Regarding MMP-9, this gelatinase seems to be responsible for IL-1 $\beta$ activation and that of other bioactive molecules, such as TNF- $\alpha$ (35) and proneurotrophins, such as pro-NGF and pro-BNDF (36), in the initial stages of the inflammatory process, while MMP. 2 is implicated in maintenance of persistent pain $(11,14)$. Further, MMP-9 may also participate in the induction, proliferation and remodeling of SGCs (37). Studies in chronic or persistent pain models indicate that communication between SGCs and sensory neurons of the ganglion are greatly increased by facilitating the formation of gap junctions between cells $(31,38)$ and by increasing sodium currents and suppressing potassium currents (39). A recent report (30) demonstrated that blockade of MMP-9 abolishes the activation of SGCs and the expression of IL-1 $\beta$. These events could be correlated with the hyperalgesia and allodynia observed in inflammatory conditions (31). Thus, persistent infusion of an MMP-9 inhibitor delays the development of mechanical allodynia in rats with spinal nerve lesions (14). One plausible explanation is that nerve injury induces a spontaneous discharge in sensory neurons releasing MMP-9 and pro-IL-1 $\beta$ into the extracellular matrix, where MMP-9 cleaves pro-IL-1 $\beta$ to IL-1 $\beta$, which promotes hyperexcitability in adjacent nociceptive neurons (16). The cleavage and activation of $\mathrm{IL}-1 \beta$ is promoted by MMP-2 in the late phase of persistent and neuropathic pain (14). In addition, MMP-2 inhibition reduced the mechanical allodynia induced by nerve injury on day 1 , but blocked this hypersensitivity on day 10 (14).

It is important to clarify that MMPs can hydrolyze other substrates and interfere in other mechanisms that are also critical for neuronal sensitization $(14,16,29)$. Thus, recent reports have demonstrated that rapid upregulation of MMP-9 in primary sensory neurons in the DRG can mask opioid analgesia without interfering in opioidinduced hyperalgesia (30). According to Berta et al. (30), it is possible that neuronal MMP-9 expression and release following acute morphine administration promote the activation of SGCs and IL-1 $\beta$, reducing the analgesic effect of the opioid.

In conclusion, to our knowledge, this is the first study to report and characterize the expression of MMPs in the TG following TMJ inflammation. The study showed that MMPs are involved in different phases during the 
development of the TMJ inflammatory response. MMP-9 is involved in the early phase, indicated by its upregulation in the TG on days 1 and 3, while MMP-2 is principally implicated in the late phase of this process, since greater expression was observed on days 7 and 10 following inflammation induced by intra-TMJ CFA administration. In addition, treatment with an MMP inhibitor attenuated increases of mechanical allodynia and orofacial hyperalgesia induced by intra-TMJ injection of CFA.

\section{References}

1. Dahlstrom L, Carlsson GE. Temporomandibular disorders and oral health-related quality of life. A systematic review. Acta Odontol Scand 2010; 68: 80-85, doi: 10.3109/ 00016350903431118.

2. Yamazaki $\mathrm{Y}$, Ren K, Shimada M, Iwata K. Modulation of paratrigeminal nociceptive neurons following temporomandibular joint inflammation in rats. Exp Neurol 2008; 214: 209-218, doi: 10.1016/j.expneurol.2008.08.005.

3. Lund JP, Lavigne G, Feine JS, Goulet JP, Chaytor DV, Sessle BJ, et al. The use of electronic devices in the diagnosis and treatment of temporomandibular disorders. $J$ Can Dent Assoc 1989; 55: 749-750.

4. Lam DK, Sessle BJ, Cairns BE, Hu JW. Neural mechanisms of temporomandibular joint and masticatory muscle pain: a possible role for peripheral glutamate receptor mechanisms. Pain Res Manag 2005; 10: 145-152.

5. Roveroni RC, Parada CA, Cecilia M, Veiga FA, Tambeli CH. Development of a behavioral model of TMJ pain in rats: the TMJ formalin test. Pain 2001; 94: 185-191, doi: 10.1016/ S0304-3959(01)00357-8.

6. Imbe H, Iwata K, Zhou QQ, Zou S, Dubner R, Ren K. Orofacial deep and cutaneous tissue inflammation and trigeminal neuronal activation. Implications for persistent temporomandibular pain. Cells Tissues Organs 2001; 169: 238-247, doi: 10.1159/000047887.

7. Sato T, Kitagawa J, Ren K, Tanaka H, Tanabe A, Watanabe $\mathrm{T}$, et al. Activation of trigeminal intranuclear pathway in rats with temporomandibular joint inflammation. J Oral Sci 2005; 47: 65-69, doi: 10.2334/josnusd.47.65.

8. Sorsa T, Tjaderhane L, Salo T. Matrix metalloproteinases (MMPs) in oral diseases. Oral Dis 2004; 10: 311-318, doi: 10.1111/j.1601-0825.2004.01038.x.

9. Krane SM. Clinical importance of metalloproteinases and their inhibitors. Ann N Y Acad Sci 1994; 732: 1-10, doi: 10.1111/j.1749-6632.1994.tb24719.x.

10. Dzwonek J, Rylski M, Kaczmarek L. Matrix metalloproteinases and their endogenous inhibitors in neuronal physiology of the adult brain. FEBS Lett 2004; 567: 129-135, doi: 10.1016/j.febslet.2004.03.070.

11. Parks WC, Wilson CL, Lopez-Boado YS. Matrix metalloproteinases as modulators of inflammation and innate immunity. Nat Rev Immunol 2004; 4: 617-629, doi: 10.1038/nri1418.

12. Khandoga A, Kessler JS, Hanschen M, Khandoga AG,

\section{Acknowledgments}

The authors would like to thank Patrícia Adriana Basile for her technical assistance. Research supported by CAPES/PROEX and FAPESP. G.C. Nascimento is the recipient of a Master's degree scholarship from FAPESP (\#2009/04430-4). R.F. Gerlach (\#2011/10336-0) and C.R.A. Leite-Panissi (\#307383/2012-1) received research grants from the CNPq.
Burggraf D, Reichel C, et al. Matrix metalloproteinase-9 promotes neutrophil and T cell recruitment and migration in the postischemic liver. J Leukoc Biol 2006; 79: 1295-1305, doi: $10.1189 / \mathrm{jlb} .0805468$.

13. Mizui $\mathrm{T}$, Ishimaru J, Miyamoto $\mathrm{K}$, Kurita K. Matrix metalloproteinase-2 in synovial lavage fluid of patients with disorders of the temporomandibular joint. $\mathrm{Br} J$ Oral Maxillofac Surg 2001; 39: 310-314, doi: 10.1054/bjom. 2001.0634

14. Kawasaki $Y, X u Z Z$, Wang $X$, Park JY, Zhuang ZY, Tan PH, et al. Distinct roles of matrix metalloproteases in the earlyand late-phase development of neuropathic pain. Nat Med 2008; 14: 331-336, doi: 10.1038/nm1723.

15. Hanani M. Satellite glial cells in sensory ganglia: from form to function. Brain Res Brain Res Rev 2005; 48: 457-476, doi: 10.1016/j.brainresrev.2004.09.001.

16. Ji RR, Xu ZZ, Wang X, Lo EH. Matrix metalloprotease regulation of neuropathic pain. Trends Pharmacol Sci 2009; 30: 336-340, doi: 10.1016/j.tips.2009.04.002.

17. Spears R, Dees LA, Sapozhnikov M, Bellinger LL, Hutchins B. Temporal changes in inflammatory mediator concentrations in an adjuvant model of temporomandibular joint inflammation. J Orofac Pain 2005; 19: 34-40.

18. Zhou Q, Imbe H, Dubner R, Ren K. Persistent Fos protein expression after orofacial deep or cutaneous tissue inflammation in rats: implications for persistent orofacial pain. $J$ Comp Neurol 1999; 412: 276-291, doi: 10.1002/(SICI)10969861(19990920)412:2<276::AID-CNE7>3.0.CO;2-9.

19. Kwan CL, Hu JW, Sessle BJ. Neuroplastic effects of neonatal capsaicin on neurons in adult rat trigeminal nucleus principalis and subnucleus oralis. J Neurophysiol 1996; 75: 298-310.

20. Bradley PP, Christensen RD, Rothstein G. Cellular and extracellular myeloperoxidase in pyogenic inflammation. Blood 1982; 60: 618-622.

21. Castro MM, Rizzi E, Figueiredo-Lopes L, Fernandes $\mathrm{K}$ Bendhack LM, Pitol DL, et al. Metalloproteinase inhibition ameliorates hypertension and prevents vascular dysfunction and remodeling in renovascular hypertensive rats. Atherosclerosis 2008; 198: 320-331, doi: 10.1016/j.athero sclerosis.2007.10.011.

22. Bradford MM. A rapid and sensitive method for the quantitation of microgram quantities of protein utilizing the principle of protein-dye binding. Anal Biochem 1976; 72: 
248-254, doi: 10.1016/0003-2697(76)90527-3.

23. Sang QX, Stetler-Stevenson WG, Liotta LA, Byers SW. Identification of type IV collagenase in rat testicular cell culture: influence of peritubular-Sertoli cell interactions. Biol Reprod 1990; 43: 956-964, doi: 10.1095/biolreprod 43.6.956.

24. Castro MM, Rizzi E, Prado CM, Rossi MA, Tanus-Santos JE, Gerlach RF. Imbalance between matrix metalloproteinases and tissue inhibitor of metalloproteinases in hypertensive vascular remodeling. Matrix Biol 2010; 29: 194-201, doi: 10.1016/j.matbio.2009.11.005.

25. Grabow TS, Dougherty PM. Cervicomedullary intrathecal injection of morphine produces antinociception in the orofacial formalin test in the rat. Anesthesiology 2001; 95: 1427-1434, doi: 10.1097/00000542-200112000-00023.

26. Harper RP, Kerins CA, Talwar R, Spears R, Hutchins B, Carlson DS, et al. Meal pattern analysis in response to temporomandibular joint inflammation in the rat. $J$ Dent Res 2000; 79: 1704-1711, doi: 10.1177/00220345000790091 101.

27. Whelan CJ. Metalloprotease inhibitors as anti-inflammatory agents: an evolving target? Curr Opin Investig Drugs 2004; 5: 511-516.

28. Schutz TC, Andersen ML, Tufik S. Sleep alterations in an experimental orofacial pain model in rats. Brain Res 2003; 993: 164-171, doi: 10.1016/j.brainres.2003.09.006.

29. Watkins LR, Maier SF. Beyond neurons: evidence that immune and glial cells contribute to pathological pain states. Physiol Rev 2002; 82: 981-1011.

30. Berta T, Liu T, Liu YC, Xu ZZ, Ji RR. Acute morphine activates satellite glial cells and up-regulates IL-1beta in dorsal root ganglia in mice via matrix metalloprotease-9. $\mathrm{Mol}$ Pain 2012; 8: 18, doi: 10.1186/1744-8069-8-18.

31. Dublin $P$, Hanani M. Satellite glial cells in sensory ganglia: their possible contribution to inflammatory pain. Brain Behav
Immun 2007; 21: 592-598, doi: 10.1016/j.bbi.2006.11.011.

32. Keast JR, Stephensen TM. Glutamate and aspartate immunoreactivity in dorsal root ganglion cells supplying visceral and somatic targets and evidence for peripheral axonal transport. J Comp Neurol 2000; 424: 577-587, doi: 10.1002/1096-9861(20000904)424:4<577::AIDCNE2>3.0.CO;2-E.

33. Candelario-Jalil E, Yang Y, Rosenberg GA. Diverse roles of matrix metalloproteinases and tissue inhibitors of metalloproteinases in neuroinflammation and cerebral ischemia. Neuroscience 2009; 158: 983-994, doi: 10.1016/j.neu roscience.2008.06.025.

34. Yong VW. Metalloproteinases: mediators of pathology and regeneration in the CNS. Nat Rev Neurosci 2005; 6: 931944, doi: 10.1038/nrn1807.

35. Cauwe B, Van den Steen PE, Opdenakker G. The biochemical, biological, and pathological kaleidoscope of cell surface substrates processed by matrix metalloproteinases. Crit Rev Biochem Mol Biol 2007; 42: 113-185, doi: 10.1080/10409230701340019.

36. Lee R, Kermani P, Teng KK, Hempstead BL. Regulation of cell survival by secreted proneurotrophins. Science 2001; 294: 1945-1948, doi: 10.1126/science.1065057.

37. Chattopadhyay S, Shubayev VI. MMP-9 controls Schwann cell proliferation and phenotypic remodeling via IGF-1 and ErbB receptor-mediated activation of MEK/ERK pathway. Glia 2009; 57: 1316-1325, doi: 10.1002/glia.20851.

38. Hanani M, Huang TY, Cherkas PS, Ledda M, Pannese E. Glial cell plasticity in sensory ganglia induced by nerve damage. Neuroscience 2002; 114: 279-283, doi: 10.1016/ S0306-4522(02)00279-8.

39. Binshtok AM, Wang H, Zimmermann K, Amaya F, Vardeh $D$, Shi L, et al. Nociceptors are interleukin-1beta sensors. $J$ Neurosci 2008; 28: 14062-14073, doi: 10.1523/ JNEUROSCI.3795-08.2008. 